\title{
Molecular-targeted therapy for elderly patients with advanced non-small cell lung cancer (Review)
}

\author{
GIOVANNA ANTONELLI ${ }^{1}$, MASSIMO LIBRA ${ }^{2}$, VINCENZO PANEBIANCO ${ }^{3}$, \\ ALESSIA ERIKA RUSSO $^{1}$, FELICE VITO VITALE ${ }^{1}$, PAOLO COLINA $^{1}$, \\ ALESSANDRO D'ANGELO ${ }^{1}$, ROSALBA ROSSELLO ${ }^{1}$ and FRANCESCO FERRA ${ }^{1}$ \\ ${ }^{1}$ Department of Medical Oncology, San Vincenzo Hospital, Taormina, Messina I-98039; \\ ${ }^{2}$ Laboratory of Translational Oncology and Functional Genomics, Section of General Pathology and Oncology, \\ Department of Biomedical Sciences, University of Catania, Catania I-95124; ${ }^{3}$ Department of Surgical Oncology, \\ San Vincenzo Hospital, Taormina, Messina I-98039, Italy
}

Received September 7, 2014; Accepted June 2, 2015

DOI: $10.3892 / \mathrm{ol} .2015 .3901$

\begin{abstract}
Lung cancer is the most common cause of cancer-related mortality in men and women. Non-small cell lung cancer (NSCLC) represents close to $90 \%$ of all lung cancers. When diagnosed, $>50 \%$ of patients are $>65$ years old. Through an improved understanding of the molecular mechanisms involved in lung oncogenesis, molecular-targeted approaches have become an essential element for the treatment of patients with NSCLC. As the toxicity profiles of the techniques are definitely more favorable compared with chemotherapy, they are particularly attractive for use in elderly patients, who are potentially more susceptible to the toxicity of systemic oncological therapies. However, studies on the activity of molecular-targeted agents in this aged patient setting are much more limited compared with those in their younger counterparts. In the present review, the literature on molecular-targeted therapy for elderly patients with advanced NSCLC is discussed. It is concluded that bevacizumab should be reserved only for highly select elderly patients with advanced NSCLC when the clinician deems it useful in the face of acceptable toxicities. In elderly patients with advanced epidermal growth factor receptor mutation-positive NSCLC, erlotinib and gefitinib appear to repeat the same favorable performance as that documented on a larger scale in the overall population of patients with activating mutations. A good toxicity profile is also confirmed for active molecules on different pathways, such as crizotinib.
\end{abstract}

Correspondence to: Dr Francesco Ferrau, Department of Medical Oncology, San Vincenzo Hospital, Via Sirina, Taormina, Messina I-98039, Italy

E-mail: francescoferrau@tin.it

Key words: elderly patients, advanced non-small cell lung cancer, molecular-targeted therapy, safety profile

\section{Contents}

1. Introduction

2. Activity of bevacizumab

3. Activity of gefitinib and erlotinib

4. Other molecular-targeted therapies

5. Ongoing clinical trials

6. Conclusion

\section{Introduction}

The 'elderly' patient with lung cancer treated with cytotoxic chemotherapy has been the subject of several studies that have assumed international importance due to sample size, methodological rigor and impact on clinical practice. Confirmed by evidence-based medicine, these important trials have led to the definition of therapeutic standards validated in this patient subset: The ELVIS study showed the superiority of vinorelbine over placebo (1); the MILES study showed a similar effectiveness between single-agent vinorelbine or gemcitabine, and single-agent efficacy over the combination of vinorelbine plus gemcitabine (2); the MILES 2P study showed better survival for cisplatin plus gemcitabine compared with cisplatin plus vinorelbine (3); the IFCT-0501 study showed survival benefits with carboplatin plus weekly paclitaxel compared with vinorelbine or gemcitabine monotherapy (4); another study showed improved survival with platinum-based adjuvant chemotherapy (5); and in a phase II trial involving elderly patients with advanced-stage small cell lung cancer, the weekly regimen of gemcitabine and docetaxel demonstrated no advantage over standard therapy (6).

The last decade of research in this field has witnessed the emergence of molecular-targeted therapies as an essential element for the treatment of patients with non-small cell lung cancer (NSCLC) (7). The studies on the activity of these novel therapies in the elderly patient with NSCLC are few $(8,9)$, comprising a more fragmented and less numerous casuistry than the experience accumulated in the younger patient $(10,11)$. The majority of significant retrospective data 
are obtained by subgroup analysis of elderly patients from the large studies performed without age restriction (12-14). To date, a lower contribution has been provided from prospective studies specifically designed to evaluate the efficacy and safety of molecular-targeted drugs in elderly patients with NSCLC (15-21).

The aim of the present review is to summarize the current data on the efficacy and safety of molecular-targeted therapy in elderly patients with advanced NSCLC.

\section{Activity of bevacizumab}

Comparing bevacizumab plus carboplatin and paclitaxel with carboplatin plus paclitaxel alone, the elderly subset analysis of the Eastern Cooperative Oncology Group (ECOG) 4599 study showed no statistically significant improvement in objective response rate (ORR), progression free survival (PFS) or overall survival (OS) in 224 patients aged $\geq 70$ years $(26 \%$ of cases). Grade III-IV adverse events were significantly more frequent in the elderly subjects compared with the younger subjects (87 vs. 61\%) (12). Another retrospective analysis was performed on 304 elderly patients aged $\geq 65$ years, out of a total of 1,430 patients enrolled in the AVAiL study, comparing the cisplatin/gemcitabine regimen alone or in combination with two different bevacizumab doses. Adding an antiangiogenic agent extended the PFS time, with no impact on survival and no significant toxicities, as in the younger patients (13).

Similarly, no significant difference was shown in the incidence and severity (grade $\geq 3$ ) of adverse events and in the outcome for elderly versus younger patients in a safety sub-analysis of bevacizumab in 623 patients aged $>65$ years who were included in the SAiL study, a phase IV international trial in a broad population of $>2,000$ patients with advanced NSCLC (22).

Therefore, conflicting retrospective data have been generated; it is possible that the following factors have led to this heterogeneity: A higher median age for the ECOG 4599 cases than the other two studies; difficulty in distinguishing between side-effects caused by bevacizumab (such as hypertension and proteinuria) and complications associated with the disease and/or age; and increased attention by clinicians on the side-effects of bevacizumab, with better management in the most recent studies (23).

The role of maintenance bevacizumab in elderly patients has also been investigated. A multicenter United States study extrapolated data on the elderly from a clinic trial that compared the efficacy and safety of pemetrexed plus carboplatin plus bevacizumab, followed by pemetrexed plus bevacizumab maintenance with paclitaxel plus carboplatin plus bevacizumab, followed by bevacizumab maintenance in patients with advanced NSCLC. There was no OS advantage in any of the age subgroups ( $\leq 70,71-75$ and $>75$ years). The pemetrexed plus bevacizumab maintenance arm exhibited significantly better PFS, but this advantage was lost in patients $>70$ years, who also suffered from increased toxicity (24).

At the 2013 American Society of Clinical Oncology Annual Meeting, Schuette et al presented a German multicenter phase III trial of pemetrexed and bevacizumab versus pemetrexed, bevacizumab and carboplatin as first-line treatment for elderly patients $\geq 65$ years with advanced non-squamous NSCLC. A total of 271 patients were enrolled and the primary endpoint was PFS. The triplet combination was superior to the doublet regimen in terms of median PFS time (6.8 vs. 4.8 months), objective response (44.4 vs. $31.4 \%$ ) and median OS time (15.2 vs. 11.6 months); in only the small group of patients with an ECOG performance status (PS) of 2, the median OS time was longer for the pemetrexed plus bevacizumab arm (11.5 vs. 3.8 months). Serious adverse events occurred with a similar incidence in the two treatment groups (48.1 vs. 49.2\%). Based on those results, the study conclusion placed emphasis on the significant impact of the triplet regimen on survival in elderly patients, with the median OS time of 15.2 months being in accordance with the most favorable results observed in this subset in the general population. The addition of carboplatin is recommended for eligible patients. However, in patients with an ECOG PS of 2, the administration of carboplatin must be carefully reviewed (25).

Despite its known toxicity, bevacizumab has been frequently used in clinical settings where the risk/benefit ratio has not been thoroughly evaluated prior to market entry and the subsequent large-scale use. This methodological problem is more clearly highlighted by the use of bevacizumab in colorectal cancer, which is the most dated indication for this drug. In subjects with lung cancer who were $>65$ years old, bevacizumab-related toxicities were observed in $28 \%$ of cases, with cardiovascular and hemorrhagic events being the most common. The elderly subset with lung cancer was more likely to have one or more contraindication to receiving bevacizumab than patients affected by other types of cancer (odds ratio, 1.7) (26). Therefore, it is necessary to extend inclusion criteria of pivotal clinical trials in order to include specific subgroups, such as elderly patients; similarly, it is important to maintain an active monitoring system in the context of pharmacovigilance programs for the detection of safety problems in the 'real world' (26).

Therefore, bevacizumab should be administered only to highly select elderly patients with advanced NSCLC when the clinician deems it useful in the face of acceptable toxicities.

\section{Activity of gefitinib and erlotinib}

Gefitinib and erlotinib are small molecule tyrosine kinase inhibitors of the epidermal growth factor receptor (EGFR), extensively studied in patients with $\operatorname{NSCLC}(27,28)$. Although the inhibitors are also used in pretreated and non-selected patients on the basis of EGFR mutational status (11,29-31), these molecules have elective indication for EGFR mutation-positive patients as front-line treatment $(32,33)$.

In a prospective phase II study, erlotinib was evaluated as first-line treatment in 80 patients $>70$ years of age with previously untreated advanced NSCLC and no selection by EGFR mutation. Erlotinib was associated with a lower incidence of toxicity compared with that observed in elderly patients treated with different chemotherapy regimens; the disease control rate of $51 \%$ was encouraging, with an ORR of $10 \%$ and disease stabilization in $41 \%$ of cases (15).

Another study with anti-EGFR therapy was performed in chemotherapy-naïve, molecularly non-selected elderly patients aged $\geq 70$ years with advanced NSCLC. This randomized phase II study (The INVITE trial) compared gefitinib with 


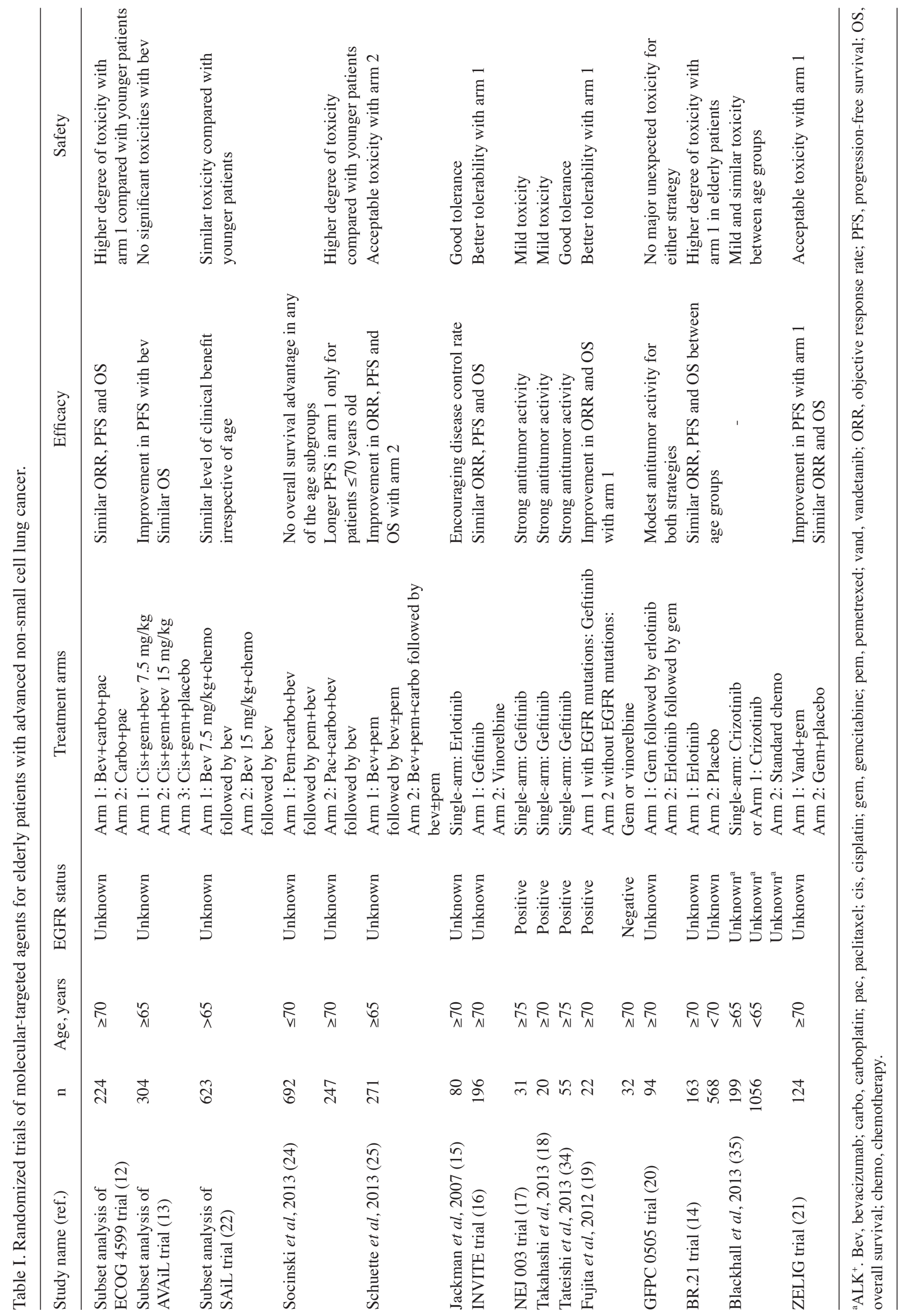


Table II. Ongoing clinical trials of molecular-targeted agents for elderly patients with advanced non-small cell lung cancer.

\begin{tabular}{|c|c|c|c|c|}
\hline $\begin{array}{l}\text { National clinical trial } \\
\text { ID numbers (ref.) }\end{array}$ & Country & Study design & Phase & Treatment arms \\
\hline NCT00678964 (36) & Germany & Randomized & II & $\begin{array}{l}\text { Arm 1: Erlotinib } \\
\text { Arm 2: Vinor+carbo }\end{array}$ \\
\hline NCT01642017 (37) & France & Non-randomized & $\mathrm{I}$ & Single-arm: Pazop \\
\hline NCT01684111 (38) & Germany & Non-randomized & $\mathrm{I}$ & Single-arm: Ninted+vinor \\
\hline NCT01683682 (39) & Germany & Non-randomized & I & Single-arm:Ninted+vinor+carbo \\
\hline NCT01077713 (40) & Italy & Randomized & II & $\begin{array}{l}\text { Arm 1: Bev+gem } \\
\text { Arm 2: Bev+gem+cis }\end{array}$ \\
\hline
\end{tabular}

Vinor, vinorelbine; carbo, carboplatin; pazop, pazopanib; ninted, nintedanib; bev, bevacizumab; cis, cisplatin.

vinorelbine as first-line treatment; activity (ORR, 3.1\% with gefitinib vs. $5.1 \%$ with vinorelbine) and efficacy parameters (PFS time of 2.7 months and OS time of 6 months for gefitinib vs. 2.9 and 8 months for vinorelbine, respectively) showed no significant differences between treatments. Grade 3 to 5 adverse events were more frequently observed in the vinorelbine arm (42\%) compared with the gefitinib arm (13\%). The merits of this trial are the perspective design specifically aimed at elderly patients and sample sizes; however it does not add relevant elements to clinical practice, such as not taking into account the EGFR mutational status (16).

The initial experiences in elderly patients with advanced EGFR mutation-positive NSCLC are beginning to be reported in the literature. A small study was performed with gefitinib as first-line treatment in 31 patients aged $\geq 75$ years with NSCLC harboring EGFR mutations. The results were quite similar to those most widely reported for the younger population, with an objective response of $74 \%$ and an overall disease control rate of $90 \%$ (17).

Limited experience was gained in a Japanese study conducted with gefitinib as first-line treatment in 20 patients aged $\geq 70$ years with non-squamous NSCLC harboring EGFR mutations. The ORR was $70 \%$, the disease control rate (complete response, partial response and stable disease) was $90 \%$ and the median PFS time was 10 months; the functional assessment of a cancer therapy-lung cancer subscale questionnaire recorded a significant improvement in the first four weeks of treatment, particularly for dyspnea and cough (18).

Another Japanese study reported a retrospective analysis of the efficacy and tolerability of first-line gefitinib in 55 NSCLC patients aged $\geq 75$ years with EGFR activating mutations. Activity (ORR, 72.7\%) and efficacy parameters (PFS, 13.8 months; OS, 29.1 months) associated with the safety profile and easy management of toxic effects confirmed the role of gefitinib as the first choice treatment for elderly patients with advanced EGFR mutation-positive NSCLC (34).

A further Japanese study confirmed the activity of gefitinib in this subset of patients, tailoring treatment according to EGFR mutation status. A total of 57 chemotherapy-naïve patients aged $\geq 70$ years with advanced NSCLC were enrolled. Of these, 3 patients were deemed ineligible. Of the remaining 54 patients, the 22 patients with EGFR mutations were treated with gefitinib, and the 32 patients without mutations received vinorelbine or gemcitabine. In this series, gefitinib showed superiority in terms of objective responses (45.5 vs. 18.8\%) and OS time (27.9 vs. 14.9 months) compared with chemotherapy; tolerability was predictably better for anti-EGFR therapy (19).

An innovative scheme for the use of erlotinib was proposed by French researchers. In one series of 97 vulnerable elderly patients with advanced NSCLC who were not selected for by EGFR expression, the activity of weekly gemcitabine followed by erlotinib at disease progression versus the reverse sequence was evaluated. Each strategy proved feasible, but with modest efficacy, producing similar results in terms of OS, and time to first and second progression (20).

The efficacy of erlotinib in second- or third-line treatment was evaluated in a retrospective analysis of 163 elderly patients with advanced NSCLC, selected from a total of 731 patients screened for the BR.21 study. Although at the expense of more severe toxicity (35 vs. 18\%), the results in terms of OS survival and quality of life were similar to those of younger patients (14).

This fact draws attention to the accuracy in the evaluation of elderly patients, even in the course of seemingly more manageable therapies such as targeted drugs. In this sense, it is instructive to report the case of an EGFR mutation-positive advanced NSCLC patient treated with first-line erlotinib, who exhibited early progression in the absence of drug-related toxicities; the medical history and pharmacokinetic study revealed co-administration of potential inducers of cytochrome P450 3A4 (in this case, fenofibrate) and a blood level of erlotinib lower than expected. Upon increasing the erlotinib dose, the tumor regression was obtained, demonstrating both how the alleged resistance was indeed drug-related interference, and the importance monitoring the use of polypharmacy, which is more frequently used in elderly patients rather than young patients (41).

In summary, these data confirm the role of gefitinib as a first choice treatment for EGFR mutation-positive advanced non-squamous NSCLC elderly patients, with a favorable toxicity profile.

\section{Other molecular-targeted therapies}

Crizotinib is an orally administered molecule that is highly active on NSCLC with EML4-ALK translocations, in the first and second line of therapy. The toxicity profile of the drug in 
elderly patients in comparison with the rest of the population ( $<65$ vs. $\geq 65$ years) was evaluated by an international multicenter study, presented at the 2013 European Society for Medical Oncology (ESMO) Annual Meeting, retrospectively analyzing data from three studies: Profile 1001 (Phase I, naive or pretreated patients), Profile 1005 (Phase II, pretreated patients) and Profile 1007 (second-line randomized vs. chemotherapy). The total sample consisted of 1,255 patients, of whom 199 (16\%) were $\geq 65$ years old. The frequency of grade III-IV adverse events associated with treatment with crizotinib was limited in absolute terms (visual disturbances, diarrhea, peripheral edema and vomiting); although the percentage was higher in patients aged $\geq 65$ years compared with those aged $<65$ years (15 vs. $7 \%$ ), this difference was not statistically significant (35).

Vandetanib is a novel orally administered molecule manifesting multikinasic inhibitory activity on EGFR, vascular endothelial growth factor (VEGF) and RET receptors, and showing activity in NSCLC when used alone or in combination with chemotherapy. The drug was recently used in an Italian study to treat 124 patients aged $>70$ years with advanced NSCLC, in combination with gemcitabine compared with gemcitabine alone (The ZELIG trial) (21). The combination of vandetanib and gemcitabine was significantly superior in terms of PFS compared with gemcitabine alone, whereas there was no difference in objective response and OS; the toxicity profile was similar between the two arms.

The aforementioned randomized trials are summarized in Table I.

\section{Ongoing clinical trials}

Several research groups are engaged in a series of clinical trials designed specifically to evaluate the role of novel molecules in this subset of patients. Examples of this, taken from browsing the CancerTrials.gov site (accessed October 2, 2013), are as follows: The role of first-line erlotinib is being compared to chemotherapy with carboplatin and vinorelbine in a randomized German study (36); a French pharmacokinetic study is evaluating the role of pazopanib in 'frail' patients (37); nintedanib dose escalation [active multikinase inhibitor of VEGF receptors (VEGFRs), platelet-derived growth factor receptor and fibroblast growth factor receptor) is being evaluated in combination with intravenous vinorelbine (38), or in combination with carboplatin and vinorelbine (39); and in an Italian study, the combination of bevacizumab and gemcitabine, with and without cisplatin is being evaluated (40). Table II summarizes the data of these ongoing clinical trials.

These trials represent just a few examples of studies specifically designed for elderly patients affected by NSCLC and treated with molecular-targeted therapy. However, further studies are required, as in the recent past, the data on elderly patients were extrapolated from numerous studies evaluating novel targeted drugs in the population with no age limit.

\section{Conclusion}

Clear expansion has been noted in the sector of molecular-targeted therapy for elderly patients with advanced NSCLC, from which a steady stream of novel data from the experience gained in clinical practice and the conclusions of ongoing studies is expected.

Based on these preliminary reports, it can be concluded that bevacizumab in elderly patients with advanced NSCLC should be reserved only for highly select patients for whom the clinician should assess the presence of a favorable risk/benefit ratio. Instead, small molecules inhibiting the EGFR pathway appear to repeat the same favorable performance in the elderly patient as that documented on a larger scale in the overall population of patients with activating mutations. The toxicity profile, definitely more favorable compared with chemotherapy, is also confirmed for active molecules on different pathways, such as crizotinib. This makes them particularly attractive for use in this group of patients who are potentially more susceptible to the toxicity of systemic oncological therapies.

\section{References}

1. No authors listed: Effects of vinorelbine on quality of life and survival of elderly patients with advanced non-small-cell lung cancer. The Elderly Lung Cancer Vinorelbine Italian Study Group. J Natl Cancer Inst 91: 66-72, 1999.

2. Gridelli C, Perrone F, Gallo C, et al: Chemotherapy for elderly patients with advanced non-small-cell lung cancer: The Multicenter Italian Lung Cancer in the Elderly Study (MILES) phase III randomized trial. J Natl Cancer Inst 95: 362-372, 2003.

3. Gridelli C, Maione P, Illiano A, et al: Cisplatin plus gemcitabine or vinorelbine for elderly patients with advanced non small-cell lung cancer: The MILES-2P studies. J Clin Oncol 25: 4663-4669, 2007.

4. Quoix E, Zalcman G, Oster JP, et al: Carboplatin and weekly paclitaxel doublet chemotherapy compared with monotherapy in elderly patients with advanced non-small-cell lung cancer: IFCT-0501 randomised, phase 3 trial. Lancet 378: 1079-1088, 2011.

5. Wisnivesky JP, Smith CB, Packer S, et al: Survival and risk of adverse events in older patients receiving postoperative adjuvant chemotherapy for resected stages II-IIIA lung cancer: Observational cohort study. BMJ 343: d4013, 2011.

6. Hainsworth JD, Carrell D, Drengler RL, et al: Weekly combination chemotherapy with docetaxel and gemcitabine as first-line treatment for elderly patients and patients with poor performance status who have extensive-stage small cell lung carcinoma: A minnie pearl cancer research network phase II trial. Cancer 100: 2437-2441, 2004.

7. Nguyen KS, Neal JW and Wakelee H: Review of the current targeted therapies for non-small-cell lung cancer. World J Clin Oncol 5: 576-587, 2014.

8. Tam TC, Ho JC, Wong MK, et al: Treatment outcomes in elderly with advanced-stage non-small cell lung cancer. Lung 191: 645-654, 2013.

9. Chen YM, Tsai CM, Fan WC, et al: Phase II randomized trial of erlotinib versus vinorelbine in chemonaive, advanced, non-small cell lung cancer patients aged 70 years or older. J Thorac Oncol 7: 412-418, 2012

10. Sandler A, Gray R, Perry MC, et al: Paclitaxel-carboplatin alone or with bevacizumab for non-small-cell lung cancer. $\mathrm{N}$ Engl J Med 355: 2542-2550, 2006.

11. Mok TS, Wu YL, Thongprasert S, et al: Gefitinib or carboplatin-paclitaxel in pulmonary adenocarcinoma. N Engl J Med 361: 947-957, 2009.

12. Ramalingam SS, Dahlberg SE, Langer CJ, Gray R, Belani CP, Brahmer JR, Sandler AB, Schiller JH and Johnson DH; Eastern Cooperative Oncology Group: Outcomes for elderly, advancedstage non-small-cell lung cancer patients treated with bevacizumab in combination with carboplatin and paclitaxel: analysis of eastern cooperative oncology group trial 4599. J Clin Oncol 26: 60-65, 2008.

13. Leighl NB, Zatloukal P, Mezger J, et al: Efficacy and safety of bevacizumab-based therapy in elderly patients with advanced or recurrent nonsquamous non-small cell lung cancer in the phase III BO17704 study (AVAiL). J Thorac Oncol 5: 1970-1976, 2010

14. Wheatley-Price P, Ding K, Seymour L, Clark GM and Shepherd FA: Erlotinib for advanced non-small-cell lung cancer in the elderly: An analysis of the national cancer institute of Canada clinical trials group study BR.21. J Clin Oncol 26: 2350-2357, 2008. 
15. Jackman DM, Yeap BY, Lindeman NI, Fidias P, Rabin MS Temel J, Skarin AT, Meyerson M, Holmes AJ, Borras AM, et al: Phase II clinical trial of chemotherapy-naive patients $>$ or $=70$ years of age treated with erlotinib for advanced non-small-cell lung cancer. J Clin Oncol 25: 760-766, 2007.

16. Crinò L,Cappuzzo F,ZatloukalP,Reck M,Pesek M, Thompson JC, Ford HE, Hirsch FR, Varella-Garcia M, Ghiorghiu S, et al: Gefitinib versus vinorelbine in chemotherapy-naive elderly patients with advanced non-small-cell lung cancer (INVITE): A randomized, phase II study. J Clin Oncol 26: 4253-4260, 2008.

17. Maemondo M, Minegishi Y, Inoue A, Kobayashi K, Harada M, Okinaga S, Morikawa N, Oizumi S, Tanaka T, Isobe H, et al: First-line gefitinib in patients aged 75 or older with advanced non-small cell lung cancer harboring epidermal growth factor receptor mutations: NEJ 003 study. J Thorac Oncol 7: 1417-1422, 2012.

18. Takahashi K, Saito H, Hasegawa Y, Ando M, Yamamoto M, Kojima E, Sugino Y, Kimura T, Yokoyama T, Ogasawara T, et al: First-line gefitinib therapy for elderly patients with non-small cell lung cancer harboring EGFR mutation: Central Japan Lung Study Group 0901. Proc IASLC: abstr P1.11-017, 2013.

19. Fujita S, Katakami N, Masago K, Yoshioka H, Tomii K, Kaneda T, Hirabayashi M, Kunimasa K, Morizane T, Mio T: Customized chemotherapy based on epidermal growth factor receptor mutation status for elderly patients with advanced non-small-cell lung cancer: A phase II trial. BMC Cancer 12: 185, 2012.

20. LeCaera H, Greillierb L, Corre R, Jullian H, Crequit J, Falchero L, Dujon C, Berard H, Vergnenegre A, Chouaid C, et al: A multicenter phase II randomized trial of gemcitabine followed by erlotinib at progression, versus the reverse sequence, in vulnerable elderly patients with advanced non small-cell lung cancer selected with a comprehensive geriatric assessment (the GFPC 0505 study). Lung Cancer 77: 97-103, 2012.

21. Gridelli C, Novello S, Zilembo N, Luciani A, Favaretto AG, De Marinis F, Genestreti G, Crinò L, Grossi F, Caffo O, et al: Phase II randomized study of vandetanib plus gemcitabine or gemcitabine plus placebo as first line treatment of advanced non small cell lung cancer in elderly patients. J Thorac Oncol 9: 733-737, 2014.

22. Garrido P, Thatcher N, Crino L, et al: Safety and efficacy of first-line bevacizumab (Bv) plus chemotherapy in elderly patients (pts) with advanced or recurrent non-squamous non-small cell lung cancer (NSCLC): SAiL (MO19390). EJC Suppl 7: 557, 2009.

23. Jatoi A: Adjuvant chemotherapy and targeted therapy in elderly non-small-cell lung cancer patients. Aging Health 8: 309-316, 2012.

24. Socinski MA, Patel JD, Garon EB, Govindan R, Reynolds CH, Spigel DR, Olsen MR, Liu J, Guba SC and Bonom P: A phase III study of pemetrexed (Pem) plus carboplatin $(\mathrm{Cb})$ plus bevacizumab (Bev) followed by maintenance pem plus bev versus paclitaxel (Pac) plus cb plus bev followed by maintenance bev in stage IIIb or IV nonsquamous non-small cell lung cancer (NS-NSCLC): Overall and age group results. J Clin Oncol 31 (suppl): abstr 8004, 2013.

25. Schuette W, Nagel S, Schneider CP, Engel-Riedel W, Schumann C, Kohlhaeufl M, Serke M, Hoeffken G, Kortsik C and Reck M; 65 plus: A randomized phase III trial of pemetrexed and bevacizumab versus pemetrexed, bevacizumab, and carboplatin as first-line treatment for elderly patients with advanced nonsquamous, non-small cell lung cancer (NSCLC). J Clin Oncol 31 (suppl): abstr 8013, 2013.

26. Hershman DL, Wright JD, Lim E, Buono DL, Tsai WY and Neugut AI: Contraindicated use of bevacizumab and toxicity in elderly patients with cancer. J Clin Oncol 31: 3592-3599, 2013.

27. Costanzo R, Piccirillo MC, Sandomenico C, et al: Gefitinib in non small cell lung cancer. J Biomed Biotechnol 2011: 815269, 2011.
28. Wang Y, Schmid-Bindert $\mathrm{G}$ and Zhou C: Erlotinib in the treatment of advanced non-small cell lung cancer: An update for clinicians. Ther Adv Med Oncol 4: 19-29, 2012.

29. Shepherd FA, Rodrigues Pereira J, Ciuleanu T, et al; National Cancer Institute of Canada Clinical Trials Group: Erlotinib in previously treated non-small-cell lung cancer. N Engl J Med 353: 123-132, 2005.

30. Thatcher N, Chang A, Parikh P, et al: Gefitinib plus best supportive care in previously treated patients with refractory advanced non-small-cell lung cancer: Results from a randomised, placebo-controlled, multicentre study (Iressa Survival Evaluation in Lung Cancer). Lancet 366: 1527-1537, 2005.

31. Kim ES, Hirsh V, Mok T, et al: Gefitinib versus docetaxel in previously treated non-small-cell lung cancer (INTEREST): A randomised phase III trial. Lancet 372: 1809-1818, 2008.

32. Mitsudomi T, Morita S, Yatabe Y, et al; West Japan Oncology Group: Gefitinib versus cisplatin plus docetaxel in patients with non-small-cell lung cancer harbouring mutations of the epidermal growth factor receptor (WJTOG3405): An open label, randomised phase 3 trial. Lancet Oncol 11: 121-128, 2010.

33. Maemondo M, Inoue A, Kobayashi K, et al; North-East Japan Study Group: Gefitinib or chemotherapy for non-small-cell lung cancer with mutated EGFR. N Engl J Med 362: 2380-2388, 2010.

34. Tateishi K, Ichiyama T, Hirai K, Agatsuma T, Koyama S, Hachiya T, Morozumi N, Shiina T and Koizumi T: Clinical outcomes in elderly patients administered gefitinib as first-line treatment in epidermal growth factor receptor-mutated non-small-cell lung cancer: Retrospective analysis in a Nagano lung cancer research group study. Med Oncol 30: 450, 2013.

35. Blackhall F, Shaw A, Jänne PA, KIM DW, Wilner KD, Schnell P, Polli S and Besse B: Crizotinib safety profile in elderly and non-elderly patients with advanced ALK+ non-small cell lung cancer. Proc IASLC: abstr P1.11-017, 2013.

36. Multicentre randomised phase II trial of erlotinib versus carboplatin/vinorelbine in elderly patients (=/> 70 years) with advanced non-small cell lung cancer). http://clinicaltrials.gov/show/NCT00678964. Accessed September 19, 2014

37. Phase I clinical and pharmacokinetic study of pazopanib in a population of frail elderly patients according SIOG criteria. http:// clinicaltrials.gov/show/NCT01642017. Accessed September 19, 2014.

38. An open label phase I dose escalation trial of oral BIBF 1120 in combination with intravenous vinorelbine in elderly patients with advanced non small lung cell cancer - stage IV (VENUS-1). http://clinicaltrials.gov/show/NCT01684111. Accessed September 19, 2014

39. An open label phase I dose escalation trial of oral BIBF 1120 in combination with intravenous carboplatin and vinorelbine in elderly patients with advanced non-small cell lung cancer - stage IV (VENUS-2). http://clinicaltrials.gov/show/NCT01683682. Accessed September 19, 2014.

40. Randomised phase II trial of bevacizumab (AVASTIN ${ }^{\circledR}$ ) in combination with gemcitabine or attenuated doses of cisplatin and gemcitabine as first-line treatment of elderly patients with advanced non-squamous non-small cell lung cancer - EAGLES http://clinicaltrials.gov/show/NCT01077713. Accessed September 19, 2014

41. Mir O, Blanchet B and Goldwasser F: Drug-induced effects on erlotinib metabolism. N Engl J Med 365: 379-380, 2011. 